\title{
DNA-DEPENDENT FORMATION
}

\section{OF THE SYNAPTINEMAL COMPLEX}

\section{A'T MEIOTIC PROPHASE}

\author{
THOMAS F. ROTH and MICHIO ITO
}

From the Department of Biology, University of California, San Diego, La Jolla, California 92038. Dr. Ito's present address is Biology Faculty of Science, Nagoya University, Nagoya, Japan

\begin{abstract}
A BSTRACT
Evalaution of microsporocytes cultured during discrete periods of meiotic prophase in the presence of deoxyadenosine, an inhibitor of DNA synthesis, indicate that: (1) late leptonema or early zygonema DNA synthesis is required to initiate the formation of the synaptinemal complex; (2) DNA synthesized during late zygonema is necessary for the disjunction of the paired homologs at diplonema; and (3) DNA synthesis in pachynema is a requisite for normal anaphase II separation of sister chromatids.
\end{abstract}

\section{INTRODUCTION}

Recently Hotta, Ito, and Stern (1) conclusively demonstrated that DNA synthesis occurs during meiotic prophase. Their data indicated that the synthesis amounted to about $0.3 \%$ of the total DNA present in cultured lily microsporocytes, and that it took place sometime during meiotic prophase. In a subsequent communication, they demonstrated that deoxyadenosine (AdR) effectively inhibited this DNA synthesis and produced various cytological abnormalities. The type of abnormality depended on the stage at which AdR was administered. Meiocytes treated in late leptonema or very early zygonema were prevented from proceeding beyond that stage. In those cells treated during early and mid-zygonema, the chromosomes remained condensed and underwent a delayed abortive first division. If AdR was administered later in synapsis, chromosome segregation often was abnormal at the second meiotic division (2).

From these observations, we reasoned that the effect of $A d R$ on pairing and disjunction might be associated with effects on the synaptinemal com- plex ( $\mathrm{SC}$ ). Since the $\mathrm{SC}$ is the only well ordered, structurally unique element thus far found between synapsed chromosomes, it seemed reasonable for us to investigate its occurrence in cells treated with deoxyadenosine during meiotic prophase.

Our results demonstrate that the formation of the SC can be prevented by the inhibition of DNA synthesis at late leptonema or early zygonema. In addition, inhibiting DNA synthesis after synapsis is begun stabilizes the pairing between chromosomes and interferes with the disjunction of the homologs which normally occur at diplonema.

\section{MATERIALS AND METHODS}

Prophase meiocytes from anthers of the lily, variety Cinnabar, were cultured in vitro essentially as described by Ito and Stern (2). In this series of experiments, the meiotic cells were cultured from each of the following bud lengths: $9 \mathrm{~mm}$ (late premeiotic), $11 \mathrm{~mm}$ (leptonema), $13 \mathrm{~mm}$ (zygonema), and $15 \mathrm{~mm}$ (pachynema). Although three buds were used for each length, the strings of meiocytes from an individual bud were cultured separately. There are a total of 24 identical strings which can be cultured since 


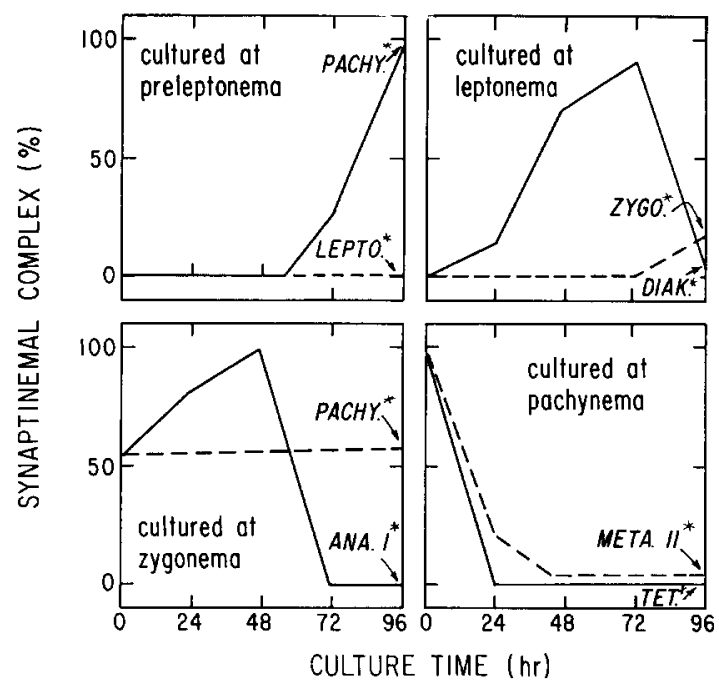

Figure 1 Effect of inhibiting DNA synthesis on the synaptinemal complex. Electron micrographs taken of sections through 80-100 nuclei from each of the four experiments were scored at the time interval indicated. In each case, a ratio was obtained between the number of condensed masses of chromatin that contained a synaptinemal complex and those that had no SC. This ratio is expressed as per cent synaptinemal complexes in the graphs shown above.

Solid lines (- $(-)$ show the approximate frequency of SC in control cells, while a dashed line (-----) indicates the frequency of $\mathrm{SC}$ in $\mathrm{AdR}$ treated cells. Since the chromosomes in most control and experimental cells were at different stages at the completion of the experiments, the meiotic stages they reach $\left({ }^{*}\right)$ are indicated in each case.

each of the six anthers in a bud has four microsporangia which contain a single string of several hundred meiocytes surrounded by sterile tissuc. At the onset of culture, we used 1 string from each bud for a chromosome squash preparation to determine the degree of synchrony and the stage of meiosis. 18 of the remaining strings were cultured in the presence of $4 \times 10^{-3}$ м 2-adenosine deoxyribonucleocide (AdR). This level of AdR gives a $60 \%$ inhibition of DNA synthesis in the lily during synapsis, whereas higher concentrations inhibit DNA synthesis almost completely (3). As a control, the remaining five strings of cells from each anther were cultured in modified White's culture medium without AdR.

At the onset of the experiment and on each of the 4 successive days, three AdR-treated strings from each anther were fixed for electron microscopy with glutaraldehyde followed by $\mathrm{OsO}_{4}$, essentially as described by Ledbetter and Porter (4). On the 4th day, we made an additional squash preparation from each anther of the AdR treated and control strings to determine the stage of meiosis at the completion of the experiment. Thin sections of the material prepared for electron microscopy were cut on a Reichert UM-2 microtome with a diamond knife. The sections were stained with a $2 \%$ aqueous solution of uranyl acetate $(\mathrm{pH} 4.9)$ for $5 \mathrm{~min}$, followed by lead citrate (5) for an equal time and viewed with a Philips 200 electron microscope.

\section{RESULTS}

\section{Normal Synaptinemal Complex}

In control cultures of microsporocytes, the synaptinemal complex (SC) first appears during zygonema, and by pachynema it is present in all cross-sections through the chromosomes. The SC follows a schedule of development similar to that reported for other organisms. Structurally, the synaptinemal complex is recognized as consisting of two thick dense lateral elements $(\sim 400 \mathrm{~A}$ thick) separated from each other by a less dense medial region $(\sim 1200 \mathrm{~A}$ wide $)$ in which lies a medial complex ( $\sim 400$ A thick). Arising from the lateral components, the chromatin appears to radiate into the surrounding nucleoplasm. A fuller description of the ultrastructure and occurrence of this structure can be found in the literature (6-8). It suffices to say here that the SC can be taken as a criterion for chromosomal pairing.

Since the formation of the SC occurs during the synaptic period, it corresponds with the interval of prophase DNA synthesis in the microsporocytes of lily. A temporal correlation thus exists between this small amount of DNA synthesis and SC. formation. In view of the similarities between synapsis in lily and other organisms, it is reasonable to suppose that this correlation between DNA synthesis and SC formation may be general in occurrence.

\section{Effect of AdR at Leptonema}

Cells placed in culture at leptonema in the presence of AdR exhibit no structures that resemble the synaptinemal complex during any of the following 4 days of culture (Fig. 1). In the absence of AdR, strings of cells cultured from the same anther proceed from leptonema through pachynema and are in diplonema or diakinesis in the same time interval (M. Ito, unpublished). However, it is, of course, difficult to define precisely the stage at which AdR treatment is initiated for any individual cell. A slight spread of develop- 
ment exists within the anther, and this is reflected in a correspondingly small spread in response to AdR treatment when the cells of a single string of cultured cells are examined under the light microscope. The results, nevertheless, clearly show that cells treated with AdR during leptonema, prior to the onset of prophase DNA synthesis, do not form synaptinemal complexes. On the other hand, those cells exposed to AdR in late leptonema or very early zygonema, at a time when DNA synthesis is initiated, may show occasional synaptinemal complexes (Fig. 2). One conclusion that may be drawn from this is that formation of the SC is inhibited under conditions in which initiation of DNA synthesis is inhibited. Moreover, once a small portion of the synaptinemal complex is formed, it remains localized on the chromosome since there is no increase in the average number of $\mathrm{SC}$ per chromatin mass during the succeeding days of culture. In such instances, the chromosomes of the cells cultured in late leptonema appear morphologically similar to chromosomes in early zygonema. The full formation of the SC thus appears to depend upon the continued synthesis of DNA during the zygotene interval.

\section{Effects of AdR at Zygonema}

Chromosomes in squash preparations shown to have initiated pairing and which exhibit a synaptinemal complex in electron micrographs respond uniformly to AdR during the 4 days of culture (Fig. 3). In all cases, the average number of SC's seen in sections through the nuclei from a given anther does not noticeably increase from day to day during the period of culture. Furthermore, the chromosomes, although only partially paired, continue to condense and appear to be in pachynema by the 4 th day. By the end of the experiment, a few of the cells have initiated cell division, but chromosome separation is abnormal. However, the majority of the cells remain in pachynema even though untreated control cells during the same period are at least in metaphase I or as far as prophase II.

From these observations of chromosome morphology during meiotic prophase, the effects of AdR appear to be relatively specific. Chromosome contraction continues in the presence of AdR, whereas SC formation does not. The specificity of AdR action is also revealed by the fact that, in its presence, cell walls continue to thicken and plasmodesmata disappear, just as they do in cells cultured in the absence of AdR. However, in those cells that initiate cell division, cross-wall formation tends to be abnormal.

\section{Effect of AdR During Pachynema}

As expected, those chromosomes that complete synapsis, and thus are in pachynema, have synaptinemal complexes embedded in most masses of chromatin (Fig. 4). Contrary to expectation, however, when these pachytene cells are placed in culture in the presence of AdR, there is no apparent delay in meiosis. These treated cells proceed through the first meiotic division at nearly the same rate as do the controls. However, most treated cells undergo an abnormal second division segregation. Thus, unlike the stabilization and inhibition of further formation of the synaptinemal complex noted in treated zygonema cells, the SC's of pachynema chromosomes remain associated with the chromosomes only for as long as they do in the corresponding control cells. It appears that the effect of $\mathrm{AdR}$ treatment at pachynema is visualized only at the later period of meiosis.

As in the case of other prophase stages subjected to AdR treatment, cell wall morphogenesis proceeds unaltered up to the initiation of crosswall formation. However, with the appearance of abnormal segregation in the second meiotic division, the cross-walls are laid down with ab normal orientation. Thus, in the case of the cell wall, AdR does not affect normal development until chromosome segregation is aberrant.

\section{ISCUSSION}

\section{DNA Synthesis and the Morphogenesis of the Synaptinemal Complex}

Ever since Moses discovered the synaptinemal complex (SC) as a unique structure associated with chromatin during synapsis, researchers have speculated on its role in meiosis (9). Its presence during pairing and its location between paired chromosomes suggested that this structure could well be involved in bringing the two homologs together in a site-for-site synapsis preparatory to crossing over. Although the mere physical presence of the SC between synapsed chromosomes is a necessary condition to implicate this structure in the process of crossing or er, it is not a sufficient one.

One of the objections which has been raised 
against assigning the $\mathrm{SC}$ a functional role in crossing over concerns the apparent absence of DNA synthesis during the interval when the $\mathrm{SC}$ is present. The fact that bulk DNA synthesis in higher organisms takes place prior to the period of synapsis and SC formation makes crossing over during prophase unlikely. However, against such objections should be placed other studies of meiotic behavior in higher organisms. Carefully timed X-irradiation of meiotic prophase cells, for example, produces abnormal segregation patterns which can best be explained by assuming that crossing over occurs during prophase (10). Thus, a fundamental conflict separates the two schools of thought on when crossing over takes place.

The finding of a small amount of DNA synthesis at the time of synapsis (1) appears to reconcile these divergent views. In addition, our demonstration that some DNA synthesis must precede the formation of the synaptinemal complex reawakens the possibility that the $\mathrm{SC}$ may be involved in site-for-site, indeed, probably in a limited DNA-for-DNA, synapsis. By this demonstration, the small burst of DNA synthesis that Hotta et al. (1) estimated to be about $0.3 \%$ of the entire genome, now becomes intimately involved with the only unique structure visible between paired homologs at the time when chromatid exchanges have been shown to take place in the lily (10). Experiments to demonstrate directly the presence of this newly synthesized DNA in the synaptinemal complex are in progress.

With this new evidence, the earlier demonstration by Coleman and Moses (7) of DNase-susceptible indium-staining nucleic acids in the synaptinemal complex takes on new importance. Their work demonstrated the presence of bulk DNA in the chromatin and lateral components of the SC. Of greater importance and directly pertinent to our present study, is that they noted a few faintly staining fibers bridging the medial zone that separates the two lateral components of the synaptinemal complex, and thus also the paired homologs. Therefore, it now seems likely that the DNA synthesized at zygonema and pachynema could well be the faint thin fibers which Coleman and Moses visualized. Indeed, they are likely to be identical with those fibers shown bridging the $\mathrm{SC}$ in Fig. 3 .

There can be little question that DNA is intimately involved in the morphogenesis of the synaptinemal complex, but what role the DNA may play in the formation of $\mathrm{SC}$ is not at all clear. It is evident that AdR inhibits DNA synthesis in lily microsporocytes, as shown by Ito et al. (3) in their studies on the reversal of AdR inhibition. They demonstrated that either the removal of AdR or the addition of thymidine ( $T d R$ ) counteracted both the block to DNA synthesis and the effect on chromosomal morphogenesis during meiotic prophase. Since both AdR and its antagonist can be shown to effect primarily DNA synthesis, we assume that the action of AdR in prophase is at the DNA level. This information and the apparent coupling of DNA synthesis and the formation of the SC strongly suggest that some DNA synthesis is required prior to the formation of the $\mathrm{SC}$. This is in no way surprising considering our present knowledge of the manner in which protein is synthesized. However, we know virtually nothing of the species of macromolecules that comprise the SC. Thus, until this information is forthcoming we can only circumstantially relate the periods of $\mathrm{SC}$ formation, DNA synthesis, and the effects of AdR inhibition to the events of meiosis.

The synthetic events of synapsis are beginning to appear increasingly complex. In regards to DNA synthesis alone, it is evident that more than a single burst of synthesis occurs during prophase.

Figure 2 Chromosomes $(C)$ from cells cultured at late leptonema in the presence of AdR for 4 days sometimes initiate pairing (arrow). The synaptinemal complex remains localized to these paired regions and is not noted in greater frequency during subsequent culture. Usually, pairing is initiated in the immediate vicinity of the cup-shaped nucleolus $(N U)$, a small portion of which is shown here. The pore-studded nuclear membrane (NM) clearly separates the nucleoplasm from the mitochondria-rich $(M)$ cytoplasm in which elements of the ribosome-studded endoplasmic reticulum $(E R)$, dictyosomes $(D)$, and lipid deposits $(L)$ are also visible. At this stage, the plasmodesmata which traverse the cell wall $(C W)$ are, for the most part, no longer present, although clear regions $(P)$ where they were are still visible in the matrix of the cell wall. $\times 8,700$. 


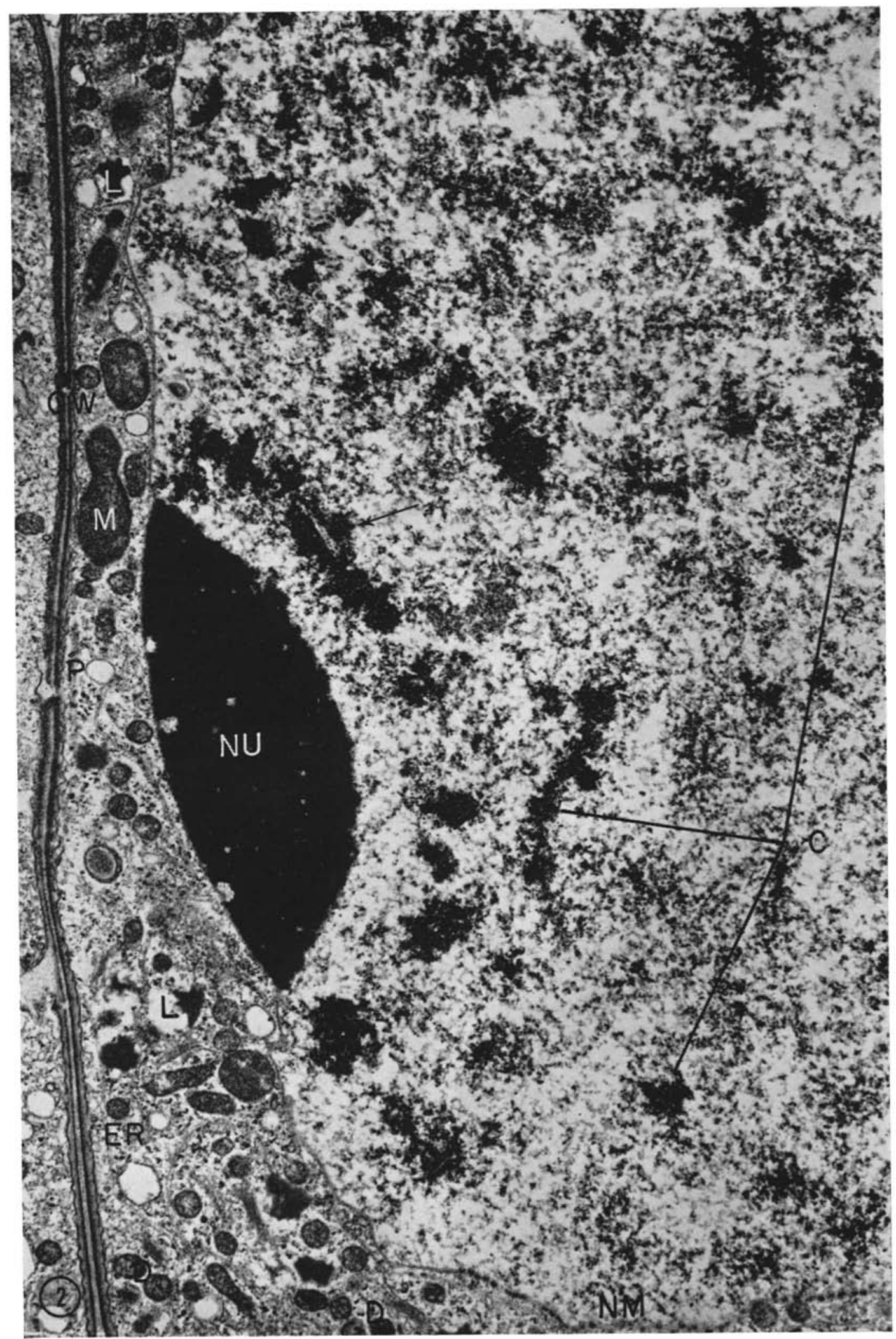

T. F. Rотн AND M. Iтo Formation of Synaptinemal Complex 


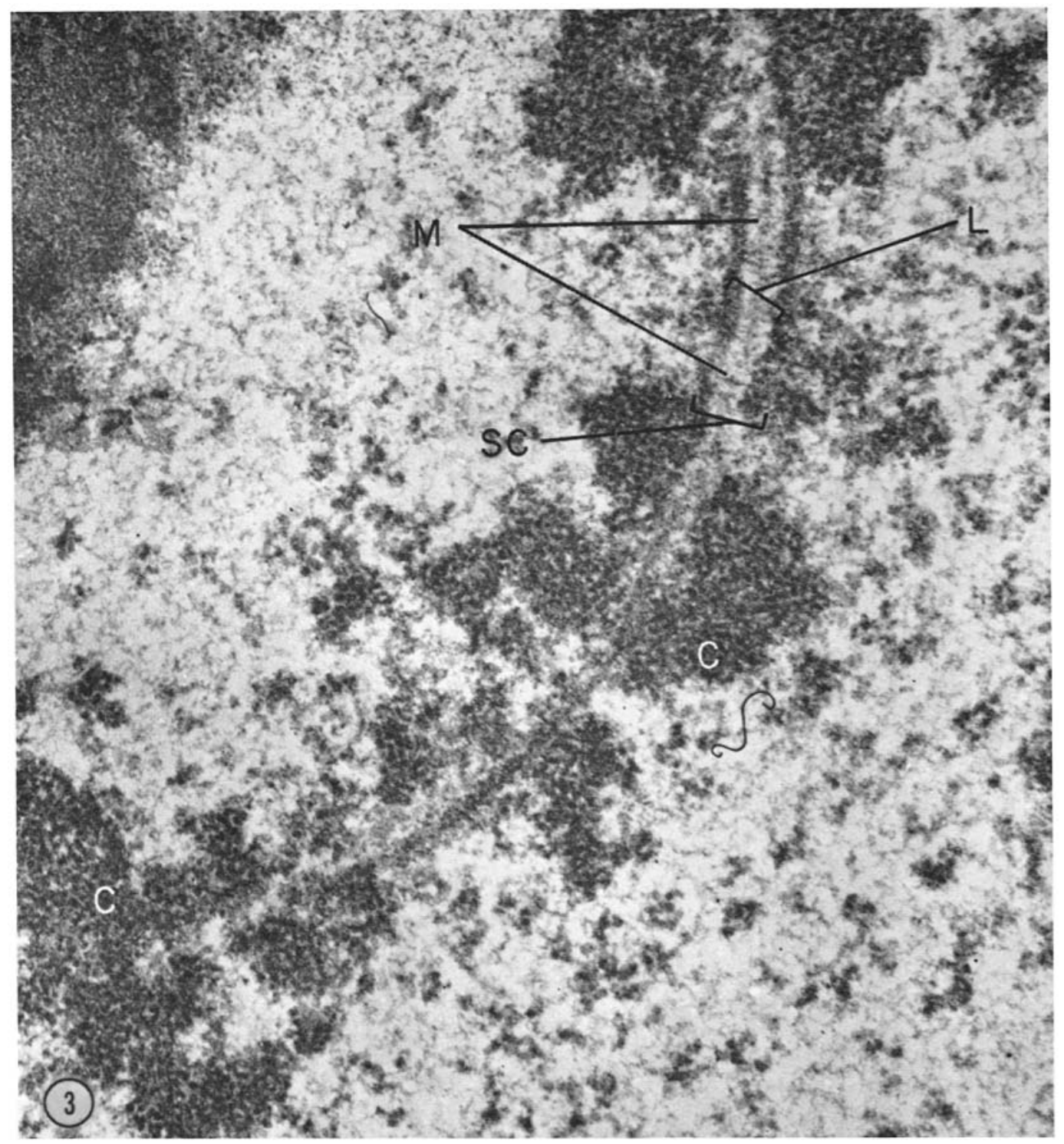

Figure 3 Once pairing is initiated, the synaptinemal complex $(S C)$ in these 3-day AdR-treated cells is noted surrounded by masses of chromatin $(C)$ at random locations throughout the nucleus. The lateral components $(L)$ of the synaptinemal complex are not easily identified except when sectioned such that both units are simultaneously visible. Bridging the gap between the lateral components, fibers appear to radiate from a slightly more dense and centrally located medial complex $(\boldsymbol{M})$.

At this zygotene stage, the chromosomes are not highly condensed and there appear to be regions in which there is little chromatin associated with the SC. AdR treatment at this time prevents subsequent disjunction of the chromosomes, which may account for the high frequency of abortive first meiotic divisions in cells treated at zygonema. $\times 44,000$.

As noted previously, some DNA synthesis must precede the formation of the synaptinemal complex. Furthermore, the blocking of DNA synthesis at zygonema stops the continued formation of the partially synthesized synaptinemal complexes. Since, in the presence of AdR, the SC becomes stabilized, further stages of chromosome develoment are inhibited or greatly slowed. It is ap- 


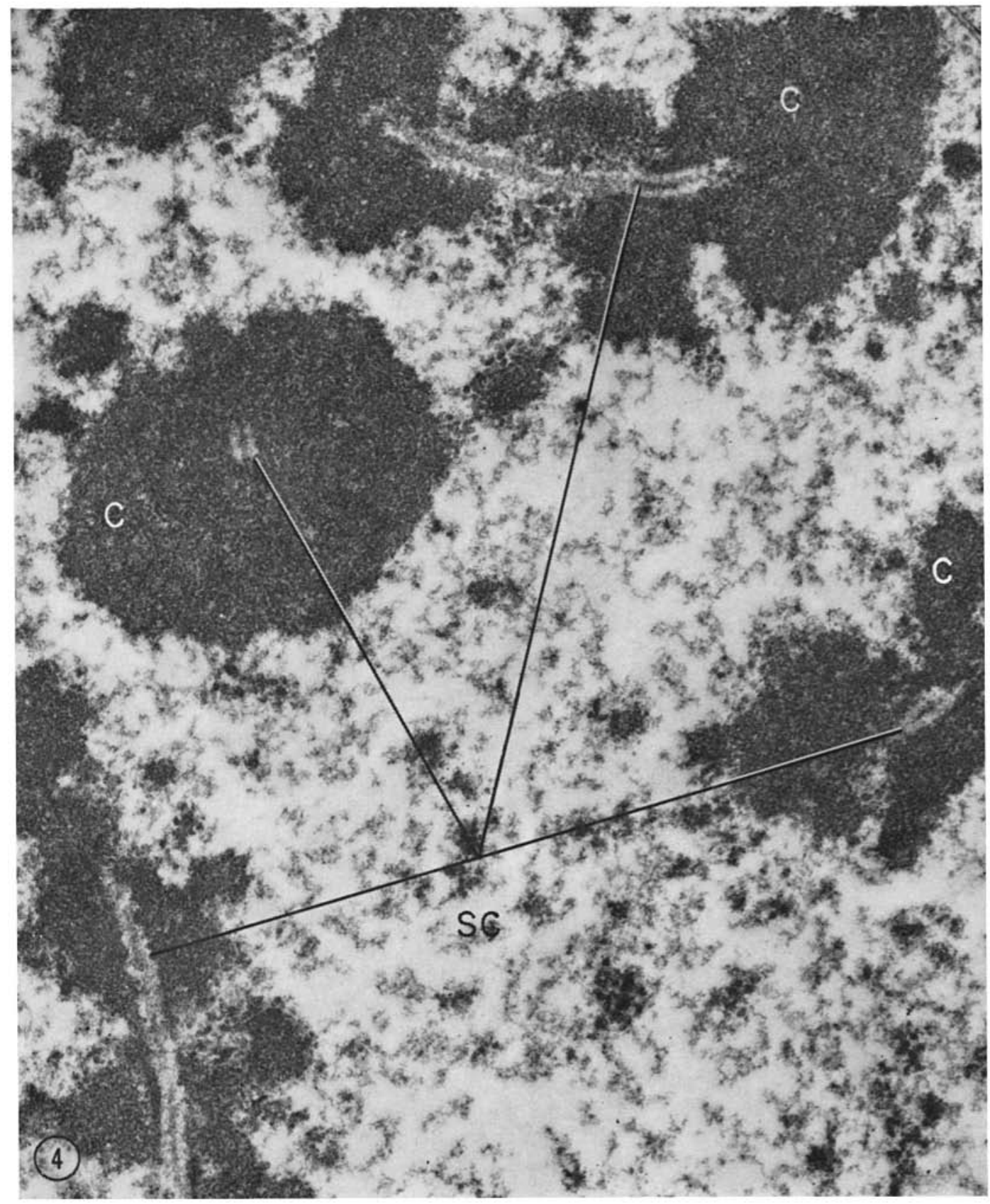

Figure 4 Upon the completion of pairing, the synaptinemal complex $(\mathrm{SC})$ is enveloped by the highly condensed chromatin $(C)$. Little or no structural difference is seen in the SC at this pachytene period when it is compared with the previous period of active synapsis. However, there is no question but that important changes have taken place. Although the AdR administered to these pachytene cells has no effect on disjunction or chromosome segregation at anaphase I, the manifestation of the DNA inhibitor is delayed until anaphase II when abnormal chromatid segregation is evinced. $\times 33,000$. 
parent, therefore, that at least two events in the maturation of the synaptinemal complex are affected by the inhibition of DNA synthesis at the synaptic period. One is the formation of the $\mathrm{SC}$ which may be related to the events which bring the two homologous chromosomes into close proximity, or perhaps to other steps such as transcription that prelude the formation of the $\mathrm{SC}$ as a morphological entity. The second event is in some way concerned with the maturation of the $\mathrm{SC}$ which permits disjunction of the homologs at diplonema.

In contrast, DNA inhibition during pachynema, after the SC appears fully formed, has no obvious effect on the ultrastructure of the SC. However, there is no question but that AdR treatment at pachynema does have a marked effect on second division segregation. In what way DNA inhibition may induce abnormal chromatid segregation is not at all clear at this time. One possibility, among several, is that although most of the DNA is replicated during the premeiotic $S$ period, a small part of the synthesis is delayed until early pachynema. It could be that this delayed synthesis is necessary to initiate events leading to the eventual separation of the sister chromatids. An equally probable possibility is that a part of the DNA synthesis at pachynema is necessary for normal centromere division. However, a more detailed analysis of the abnormal second meiotic division must be completed before speculation of this type can be taken seriously. Certainly, an analysis of centromere replication similar to that carried out by Luykx (11) may be helpful in resolving this question.

It is evident that a minimum of three distinct periods of DNA synthesis are delineated by the three morphological effects just discussed. In this regard, it is of importance that Hotta, Ito, and

\section{B I B L I O G R A P H Y}

1. Hotta, Y., M. Ito, and H. Stern. 1966. Synthesis of DNA during meiosis. Proc. Natl. Acad. Sci. 56:1184.

2. Ito, M., and H. Stern. 1967. Studies of meiosis in vitro. I. In vitro culture of meiotic cells. Develop. Biol. 16:34.

3. Iтo, M., Y. Нотta, and H. Sirern. 1967. Studies of meiosis in vitro. II. Effect of inhibiting DNA synthesis during meiotic prophase on chromosome structure and behavior. Develop. Biol. 16:54.
Stern were able to demonstrate the synthesis of at least three, or possibly four, classes of DNA that differed in relative amounts at different times during the prophase interval (1). Of real interest is the possibility of directly relating the morphologically distinct developmental changes we described with the classes of DNA they detected. However, it is evident that it is not possible to accomplish this correlation until more is known about the localization of the molecules that constitute the synaptinemal complex. Until that time, we must infer from other studies the role that DNA may have in initiating a series of biochemical events leading to the formation of the synaptinemal complex. An attempt to define more precisely the chemical composition of the $\mathrm{SC}$ is in progress.

In summary, we conclude that: (1) a late leptonema or early zygonema period of DNA synthesis is required to initiate the formation of the synaptinemal complex; (2) a distinct class of DNA is synthesized during late zygonema which is necessary for the disjunction of the paired homologs at diplonema; and (3) DNA synthesis in pachynema is required for normal anaphase II separation of sister chromatids.

We are indebted to Dr. H. Stern for his interest in and critical discussion of both the experiments and the manuscript. We also thank Mrs. Sarita Beveridge for her technical assistance in various aspects of the electron microscopy. This work was supported by a National Science Foundation Grant GB-5173X and a National Institutes of Health Grant USPHS-GM12072.

This work was reported in a preliminary form at the Society for Cell Biology meeting in Houston, Texas (J. Cell Biol. 1966. 31:96A (Abstr.)).

Received for publication 10 March 1967; revision accepted 8 June 1967.
4. Ledhetter, M. C., and K. R. Porter. 1963. A "microtubule" in plant cell fine structure. $J$. Cell Biol. 19:239.

5. Venable, J. H., and R. Coggeshall. 1965. $\Lambda$ simplified lead citrate stain for electron microscopy. J. Cell Biol. 25:407.

6. Moses, M. J. 1964. The nucleus and chromosomes: $\Lambda$ cytological perspective. In: Cytology and Cell Physiology. G. Bourne, editor. Aca demic Press Inc., New York. 423. 
7. Coleman, J. R., and M. J. Moses. 1964. DNA and the fine structure of synaptic chromosomes in the domestic rooster (Gallus domesticus). J. Cell Biol. 23:63.

8. Rotr, T. F. 1966. Changes in the synaptinemal complex during meiotic prophase in mosquito oocytes. Protoplasma. 61:346.

9. Moses, M. J. 1956. Chromosomal structures in crayfish spermatocytes. J. Biophys. Biochem. Cytol. 2:215.

10. Mrtra, S. 1958. Effects of X-rays on chromosomes of Lilium longiflorum during meiosis. Genetics. 43:771.

11. LuYkx, P. 1965. The structure of kinetochore in meiosis and mitosis in Urechis eggs. Exptl. Cell Res. 39:643. 